\title{
Prediction model for concentration measurement error based on SVR in the NDIR sensor
}

\author{
Wenjuan Cheng, Shixiang Tang, Yongzhong Wu \\ School of Computer and Information \\ Hefei University of Technology \\ Hefei, Anhui, China \\ cheng@ah.edu.cn
}

\begin{abstract}
Affected by internal and external mechanical system, environment and other factors, which most likely cause lower measuring precision that can not reach the requirements of measurement and control system in present NDIR sensor. To deal with this problem, this paper puts forward the method of building model for concentration measurement error based on SVR: based on using the differential absorption detection technology to eliminate mechanical factors inside sensor, it adopt SVR learning algorithm, which can build concentration measurement error prediction model under dynamic environment in the given training set. Then we can make corresponding error compensation for gas concentration measurement by using the model. So the gas measurement concentration is largely close to the true value. The experimental result show that regression effect of the SVR learning algorithm is good for model of concentration measurement error, meet the demand of precision of measurement and control system.
\end{abstract}

Keywords-NDIR sensor; the differential absorption detection technology; SVR; concentration measurement error mode

\section{INTRODUCTION}

In recent years, the Non-dispersed Infrared technology[1] has been widely used because of its high stability, sensitivity and larger measuring range. However, it can perform well only in a relatively stable environment (such as temperature, humidity, air-pressure, dust, etc.), whose main detection principle is Lambert Beer's Law. Large error emerges as measurement value of gas concentration deviate from the true value which caused by obvious environmental changes. In order to improve the measurement precision in dynamic environment, some researchers abandon the Lambert Beer's Law, turning to use a learning algorithm such as multiple regression analysis, neural network[2], the SVR[3] (support vector regression machine) to fit the density of measured gas in a changing environment. But problem comes when using the concentration-model according to the above methods: on a given training set, it performances well, but in the process of predicting concentration of measured gas, it works bad with the failure that the measurement precision often could not reach the requirement of measurement and control system. The main reason is that no leverage existing Lambert Beer's Law to ensure that: in a changing environment, the gas concentration measured by the sensor close to the real value partly, although this level cannot meet the requirements of system.

Therefore, using Lambert Beer's Law and differential absorption detection technique to eliminate internal mechanical factors of sensor, make the measured gas concentration close to the real value partly, and then take the SVR learning algorithm in a given training set to fit the nonlinear relationship model between measurement error and the factors such as the temperature, humidity, pressure. The model is a prediction model for concentration measurement error, which can estimate the measurement error of sensors according to known temperature, pressure, humidity. Then, the gas measurement concentration is largely close to the true value with making the measurement error as compensation to concentration measured by the sensor, to meet the demand of measurement precision of measurement and control system.

\section{DIFFERENTIAL ABSORPTION DETECTION PRINCIPLE AND ITS PROBLEMS}

Common NDIR sensor consists of thermal infrared light source, gas chamber, infrared detector with two narrowband filters .The sample graph in figure.1. When the infrared radiation passes through for the gas in the chamber, the gas molecules absorb light energy if the absorption spectrum of the gas within the scope of the incident spectrum, then intensity attenuation will happen in the corresponding spectral line intensity attenuation. The attenuation degree follows the Lambert Beer's Law.

The differential absorption detection technique based on double wavelength and single photon, whose principle is shown in figure.2, can be used to eliminate other interfering gases of sensor, instable output power of light source and photoelectric device's time drift, temperature drift and other internal factors which can affect measurement of gas concentration. It often adopts the double wavelength single photon difference absorption detection technology.

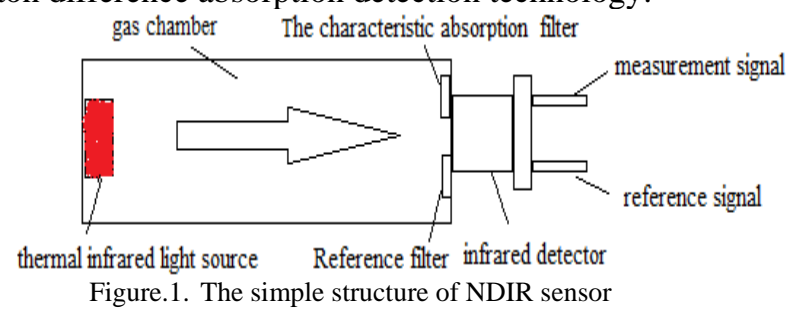




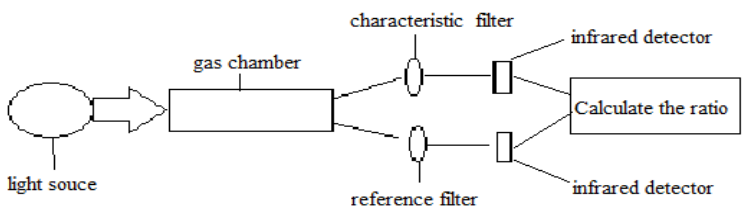

Figure.2. Differential absorption detection principle

The wavelength of gas characteristic filter equals $\lambda_{\text {gas }}$, locating in the characteristic absorption peaks of measured gas. The wavelength of referenced filter equals $\lambda_{\text {ref, which is }}$ weakly absorbed by measured gas. According to the Lambert Beer's Law, and considering other interfering gases of sensor, instable output power of light source and photoelectric device's time drift, temperature drift, here has:

$$
\begin{gathered}
I_{\text {gas }}^{\prime}=I_{\text {gas }} \exp \left(-\alpha_{\text {gas }} C l-\beta_{\text {gas }}\right) \\
I_{\text {ref }}^{\prime}=I_{\text {ref }} \exp \left(-\alpha_{\text {ref }} C l-\beta_{\text {ref }}\right)
\end{gathered}
$$

The $I_{\text {gas }}$ and $I_{\text {gas }}$ are respectively light intensity before and after the light with wavelength of $\lambda_{\text {gas }}$ passing the absorbing chamber, $\mathrm{I}_{\text {ref }}$ and $\mathrm{I}_{\text {ref }}$ are the same as $\mathrm{I}_{\text {gas }}$ and $\mathrm{I}_{\text {gas }}$ for $\lambda_{\text {ref. }}$ It is easy to know $\mathrm{I}_{\text {gas }} \approx \mathrm{I}_{\text {ref. }}$. The $\beta_{\text {gas }}$ and $\beta_{\text {ref }}$ represent the various internal factors have been talked above respectively, it is easy to know $\beta_{\text {gas }} \approx \beta_{\text {ref. }}$. When the wavelength of the light equals $\lambda_{\text {gas }}$, the absorption coefficient of measured gas ia $\alpha_{\text {gas }}$. The $\alpha_{\text {ref }}$ is the same as $\alpha_{\text {gas }}$ for $\lambda_{\text {ref }}$. Because of the weak absorption of measured gas, so $\alpha_{\text {ref }} \approx 0$ can be reasonable. Here $C$ represents unknown concentration of measured gas, $l$ represents the optical path when the light pass the absorption chamber. In conclusion, the (1) divided by (2), here gets:

$$
C=\frac{1}{\alpha_{\text {gas }} l} \ln \left(\frac{I_{\text {ref }}^{\prime}}{I_{\text {gas }}^{\prime}}\right)
$$

Equation (3) contains no influence coefficient of internal mechanical factors, so the difference detection technology eliminates effect of various uncertain mechanical factors. But it does not solve the influence of environmental factors such as temperature, pressure. The main reason is that the $\alpha_{\text {gas }}$ is assumed to be a fixed value. In fact, the $\alpha_{\text {gas }}$ is not only related to the wavelength of the $\lambda_{\text {gas }}$, but also related with temperature, pressure. When the surrounding environment changes apparently, large error will emerge as using the (3).Thus measurement precision cannot meet the requirement of measurement and control system. Since the concentration measurement error of the sensor affects by environmental factors such as temperature, pressure, humidity. It may be alright to find out the complex nonlinear relationship between error and various environmental factors - that is the error prediction model. The SVR learning algorithm can give a solution.

\section{PREDICTION MODEL FOR CONCENTRATION MEASUREMENT ERROR}

The SVR (Support Vector Regression) is essentially a regression problem which using a given training set $T$ consists of $\mathrm{m}(\mathrm{m}>0)$ samples:

$$
\begin{aligned}
& T=\left\{\left(\vec{x}^{(1)}, y^{(1)}\right), \cdots,\left(\vec{x}^{(m)}, y^{(m)}\right)\right\} \\
& \vec{x}^{(i)} \in R^{(n)}, y^{(i)} \in R,(1 \leq i \leq m), n>0
\end{aligned}
$$

Then find the unknown function relationship between independent variables $\vec{x}$ and y:

$$
f: y=f(\vec{x}, \vec{\theta}) \quad \vec{\theta} \in R^{(n)}
$$

Where $\vec{\theta}$ is the unknown parameter vector, $\vec{X}$ is the vector composed of the elements related to $y$. For a given training set, proper training methods can be used, such as the least square method, to get the value of $\vec{\theta}$, the corresponding value of y for the given $\vec{x}$.

The basic idea of SVR is that it introduces the insensitive loss function, which is limited to linear function set:

$$
f(\vec{x})=\vec{\omega} \cdot \vec{x}+b \quad \vec{\omega} \in R^{(n)}, b \in R
$$

and estimates the regression function in (6) on the basis of using SRM (structural risk minimization) principle. We can get an original optimal problem. Its form shows below:

$$
\begin{array}{ll}
\min _{\vec{\omega}, b, \xi, \xi^{*}} & \frac{1}{2}\|\vec{\omega}\|^{2}+c \sum_{i=1}^{m} \xi^{(i)}+c \sum_{i=1}^{m} \xi^{(i)} \\
\text { s.t. } & \left(\vec{\omega} \cdot \vec{x}^{(i)}+b\right)-y^{(i)} \leq \varepsilon+\xi^{(i)}, 1 \leq i \leq m \\
& y^{(i)}-\left(\vec{\omega} \cdot \vec{x}^{(i)}+b\right) \leq \varepsilon+\xi^{(i)}, 1 \leq i \leq m \\
& \xi^{(i)}, \xi^{(i)} \geq 0,1 \leq i \leq m
\end{array}
$$

Where $\|\omega\|^{2}$ is the confidence limit, which reflects expression ability of function set. $\sum_{i=1}^{m}\left(\xi^{(i)}+\xi^{*(i)}\right)$ represents the empirical risk. ${ }^{C}$ is penalty factor, it balances the confidence limit against empirical risk. Minimizing the weighted sum of the two reflects the principle of structural risk minimization. $\xi^{(i)}, \xi^{\xi^{(i)}}$ are slack variables, its value marks out the corresponding points near or far from the group. Like SVM(support vector machine), in order to use kernel functions to deal with samples of the nonlinear space, SVR usually use Lagrange Duality Theorem and KKT conditions to solve the dual problem instead of directly solving the original problem. Finally, SVR approach yields a decision function of the form:

$$
f(\vec{x})=\sum_{i=1}^{m}\left(\alpha^{(i)}-\alpha^{*(i)}\right) K\left(\vec{x}, \vec{x}^{(i)}\right)+b \quad 0 \leq \alpha^{(i)}, \alpha^{*(i)} \leq c
$$

Where $\alpha^{(i)}, \alpha^{*(i)}$ are the Lagrange coefficients, $K\left(\vec{x}, \vec{x}^{(i)}\right)$ is the kernel function[4]. The RBF kernel function is usually be chosen with its form showed as below:

$$
K\left(\vec{x}, \vec{x}^{(i)}\right)=\exp \left(-\frac{\left\|\vec{x}-\vec{x}^{(i)}\right\|^{2}}{2 \sigma^{2}}\right)
$$

The above parameters can be acquired through the study of the training set. According to the decision function and the 
given $\vec{x}$, the value of y can be predicted. The performance and the effect of SVR are related to the punish coefficient $C$ and the kernel function parameters $\sigma$.Usually, they can be selected by K-fold cross validation (K usually take 5 or 10) or Swarm intelligence algorithm, such as glowworm optimization algorithm[5], particle swarm optimization algorithm and so on.

The prediction model for concentration measurement error builds on the basis of SVR. With running the NDIR sensor system in different environments for a period of time, then we take 60 groups of history sample data of measurement and control system. For each group of data, temperature, pressure, humidity, the real and measured value of gas concentration are constant. Choosing 30 groups as the training set, the dependent variable $\mathrm{y}$ is the difference between real concentration and the measured concentration for each. Here, $\mathrm{y}$ is the gas concentration measurement error discussed above. And the independent variable $\vec{X}$ is a 3dimensional vector consisting of the temperature, pressure, humidity elements of each. The main task of the training set is taking the value of $\vec{x}$ and $\mathrm{y}$ into the SVR, then the decision function is acquired, which is just the prediction model for the concentration measurement error. It shows the complex nonlinear relationship between the value of concentration measurement error with temperature, pressure, humidity.

In addition, the other 30 groups work as the test set and test the accuracy of the prediction model. With the corresponding $\vec{X}$ of each set of data in the test set taking into the model, the sensor's measurement error can be predicted. Then, the error can be used as a compensation for the measured concentration values of the sensor. The accuracy of the model will be known when comparing the ultimate concentration value with the real value of gas concentration.

\section{THE EXPERIMENTAL RESULTS AND ANALYSIS}

A measurement and control system is built in the lab in order to simulate a common environment (with: temperature comes from- $10{ }^{\circ} \mathrm{C}$ to $50{ }^{\circ} \mathrm{C}$, pressure is101.325 Kpa, humidity belongs $0 \sim 100 \%$ RH.) where the sensor may be in. The density of test gas is $1 \%$ to $5 \%$. In figure.3, it shows the comparison of the gas density gained from the prediction model for concentration measurement error that based on SVR, the true density and the density gained without the model. The horizontal axis represents the 30 samples of the test sets, vertical axis is the corresponding gas concentration. It is easy to see, the model can far approach to the true gas concentration.

The 30 samples of the test set can be divided into 5 groups according to different gas concentration, as is showed in Table 1.Each group separately contains the true gas concentration, concentration gained from the model and the average value of the measurement concentration. The error of the model is less than $1 \%$ after calculating and comparing, which can satisfy the requirement of most of the measurement and control system.

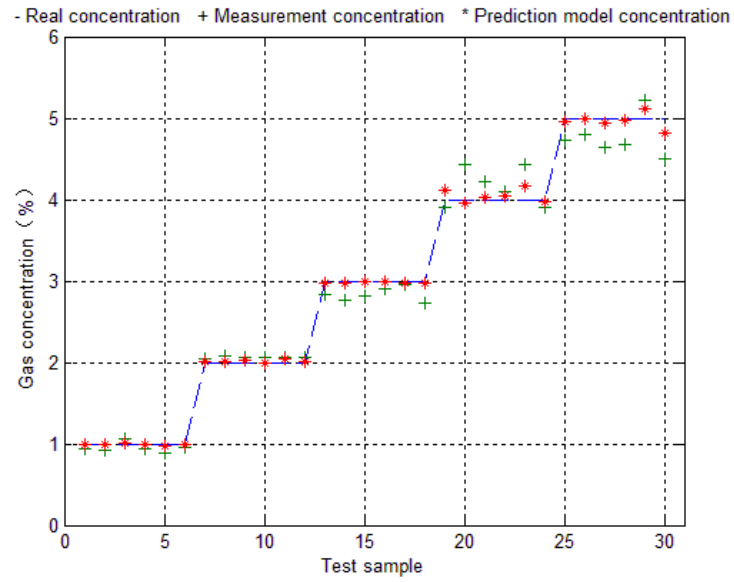

Figure.3. Comparison of real concentration, measured concent ration, and prediction model concentration

Table 1 The error of model

\begin{tabular}{|c|c|c|c|}
\hline $\begin{array}{c}\text { real } \\
\text { concentration } \\
(\%)\end{array}$ & $\begin{array}{c}\text { measured } \\
\text { concentration } \\
(\%)\end{array}$ & $\begin{array}{c}\text { prediction } \\
\text { model } \\
\text { concentration } \\
(\%)\end{array}$ & Model error \\
\hline 1 & 0.948 & 0.992 & $0.8 \%$ \\
\hline 2 & 2.615 & 2.014 & $0.695 \%$ \\
\hline 3 & 2.835 & 2.981 & $0.65 \%$ \\
\hline 4 & 4.167 & 4.037 & $0.90 \%$ \\
\hline 5 & 4.7615 & 4.961 & $0.776 \%$ \\
\hline
\end{tabular}

\section{CONCLUSION}

Because of larger changes in environmental factors, the present NDIR sensor cannot reach the requirement of measurement and control system about the measurement precision. To solve the problem, differential absorption detection technique can be used to eliminate the effect of the sensor's internal factors. Then we take the SVR learning algorithm to establish the prediction model for concentration measurement error in a dynamic changing environment. With the model, concentration measurement error can be predicted in the real-time measurement of sensor. Adding the error to the measured value as compensation, the final measured value of concentration of the sensor can meet the demand of accuracy of the measurement and control system. 


\section{Acknowledgement}

In this paper, the research was sponsored by the National Natural Science Foundation of China (Project No. 51274078) and the Fundamental Research Funds for the Central Universities (Project No. JZ2014HGBZ0009).

\section{REFERENCES}

[1] M. Arndt and M. Sauer. Spectroscopic carbon dioxide sensor for automotive applications. In Proceedings of the IEEE Sensors 2004, Vienna, vol. 1, pp. 252-255, Oct. 2004.

[2] Q.J. Wang and J.L. Cheng. Forecast of coal mine gas concentration based on the immune neural network model. Journal of China Coal Society,vol. 33,pp. 665-669,2008.

[3] V. N. Vapnik. The Nature of Statistical Learning Theory. SpringerVerlag, New York, 1995.

[4] A. J. Smola and B. Scholkopf. Lerning with Kernels. MIT Press, Cambridge, Massachusetts, 2002.

[5] K.F. Huang and Z.G. Liu. Gas sensor fault diagnosis based on ASGSO-SVR. Journal of China Coal Society,vol. 38,pp. 518523,Sep.2013. 